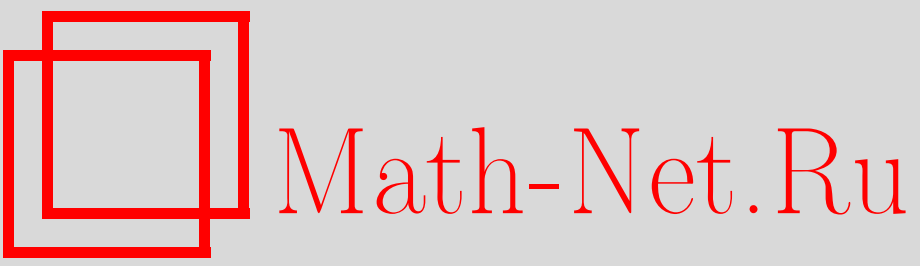

А. Л. Сараев, K теории фазовых превращений в композиционных материалах с нестабильными компонентами, Вестн. Сам. гос. техн. ун-та. Сер. Физ.-мат. науки, 2007, выпуск 2(), 85-89

DOI: https://doi.org/10.14498/vsgtu534

Использование Общероссийского математического портала Math-Net.Ru подразумевает, что вы прочитали и согласны с пользовательским соглашением http://www.mathnet.ru/rus/agreement

Параметры загрузки:

IP: 3.80 .253 .173

26 апреля 2023 г., 08:34:40 


\section{УДК 539.4}

\section{А. Л. Сараев \\ К ТЕОРИИ ФАЗОВЫХ ПРЕВРАЩЕНИЙ В КОМПОЗИЦИОННЫХ МАТЕРИАЛАХ С НЕСТАБИЛЬНЫМИ КОМПОНЕНТАМИ}

Представленъ математические модели фбазовых превращений в нестабилъных компонентах композиционных материалов. Статистическое осреднение нелинейнъх систем уравнений равновесия микронеоднородных сред с нестабилъныли компонентами позволило установить макроскопические определяющие уравнения их деббормирования и вычислить соответствуюшие эбббективнъе характеристики.

1. Пусть двухкомпонентный композиционный материал занимает объём $V$, ограниченный поверхностью $S$. Первый компонент $V_{1}$ образует связующую матрицу, второй компонент $V_{2}-$ представляет собой отдельные сферические включения. Фазовый переход первого рода происходит в объёме включений $V_{2}$. Объём зарождающейся и развивающейся новой фазы внутри включений обозначим $V_{p}$, объем старой фазы $-V_{q},\left(V_{p}+V_{q}=V_{2}\right)$. При фразовом превращении $\left(V_{p} \rightarrow V_{q}\right)$ в материале новой фазы под воздействием внешних нагрузок возникают и развиваются необратимые структурные деформации $\alpha_{i j}(\boldsymbol{r})$, вызванные перестройкой кристаллической и доменной структуры материала. Эти деформации являются ограниченными предельными сдвигами двойниковых доменов $0 \leqslant \alpha=\sqrt{\alpha_{i j} \alpha_{i j}} \leqslant \alpha_{\max }$, где $\alpha_{\max }-$ известный максимальный уровень структурных деформаций. Закон Гука такой среды имеет вид [1]:

$$
\begin{array}{ll}
\sigma_{i j}=2 \mu_{1} \varepsilon_{i j}+\delta_{i j} \lambda_{1} \varepsilon_{k k} & \left(\boldsymbol{r} \in V_{1}\right), \\
\sigma_{i j}=2 \mu_{2} \varepsilon_{i j}+\delta_{i j} \lambda_{2} \varepsilon_{k k} & \left(\boldsymbol{r} \in V_{q}\right), \\
\sigma_{i j}=2 \mu_{p}\left(\varepsilon_{i j}-\alpha_{i j}\right)+\delta_{i j} \lambda_{p} \varepsilon_{k k} & \left(\boldsymbol{r} \in V_{p}\right) .
\end{array}
$$

Здесь $\sigma_{i j}, \varepsilon_{i j}$ - тензоры напряжений и полных деформаций; $\mu_{s}, \lambda_{s}(s=1,2,3)$ - параметры Ламе компонентов.

В качестве условий прямого и обратного фазовых принимаются поверхности нагружения:

$$
\begin{array}{ll}
s_{i j} s_{i j}=k_{+}{ }^{2}(\alpha) & \left(V_{p} \rightarrow V_{q}\right), \\
s_{i j} s_{i j}=k_{-}{ }^{2}(\alpha) & \left(V_{q} \rightarrow V_{p}\right) .
\end{array}
$$

где $k_{+,-}=k_{+,-}^{\infty}+n_{+,-}^{\infty} \cdot \alpha+\left(\left(k_{+,-}^{0}-k_{+,-}^{\infty}\right)+\left(n_{+,-}^{0}-n_{+,-}^{\infty}\right) \cdot \alpha\right)\left(1-\exp \left(\frac{n_{+,-}^{0}-n_{+,-}^{\infty}}{k_{+,-}^{0}-k_{+,-}^{\infty}} \cdot \alpha\right)\right) \cdot$ Здесь $k_{+,-}, n_{+,-}-$параметры упрочнения, характеризующие скорость перемещения поверхностей (2) в шестимерном пространстве напряжений. Эти параметры зависят от температуры и их значения определяют тип поведения нестабильной среды (сверхупругость, эффект «памяти формы» или обычное пластическое течение), при этом параметрам $k_{+}, n_{+}$соответствует прямой фазовый переход, параметрам $k_{-}, n_{-}-$обратный.

Геометрические особенности такой фазовой структуры описываются набором случайных изотропных индикаторных функций координат $\kappa_{s}(\boldsymbol{r})(s=1, p, q)$, каждая из которых равна единице в точках объёма $V_{s}$ и равна нулю вне этого объёма.

С помощью этих функций локальный закон Гука для среды записывается в виде:

$$
\begin{aligned}
& s_{i j}(\boldsymbol{r})=2\left(\mu_{1}+\left(\mu_{2}-\mu_{1}\right) \kappa_{q}(\boldsymbol{r})+\left(\mu_{p}-\mu_{2}\right) \kappa_{p}(\boldsymbol{r})\right) e_{i j}(\boldsymbol{r})-2 \mu_{p} \kappa_{p}(\boldsymbol{r}) \alpha_{i j}(\boldsymbol{r}), \\
& \sigma_{k k}(\boldsymbol{r})=3\left(K_{1}+\left(K_{2}-K_{1}\right) \kappa_{q}(\boldsymbol{r})+\left(K_{p}-K_{2}\right) \kappa_{p}(\boldsymbol{r})\right) \varepsilon_{k k}(\boldsymbol{r}) .
\end{aligned}
$$

Здесь $s_{i j}=\sigma_{k k}-\frac{1}{3} \delta_{i j} \cdot \sigma_{i j}, e_{i j}=\varepsilon_{i j}-\frac{1}{3} \sigma_{i j} \varepsilon_{k k}, K_{s}=\frac{2}{3} \mu_{s}+\lambda_{s}$, структурные деформации удовлетворяют условию несжимаемости $\alpha_{k k}(r)=0$.

Индикаторные фонкции, напряжения, полные и структурные деформации предполагаются статистически однородными и эргодическими полями, поэтому их математические ожидания совпадают со средними значениями по полному объёму $V$ и объёмам фраз $V_{s}[2]$ :

$$
\langle f\rangle=\frac{1}{V} \int_{V} f(\boldsymbol{r}) d \boldsymbol{r}, \quad\langle f\rangle_{s}=\frac{1}{V_{s}} \int_{V_{s}} f(\boldsymbol{r}) d \boldsymbol{r} \quad(s=1,2, p, q),
$$

где угловыми скобками обозначена операция осреднения. 
Для установления макроскопических определяющих уравнений материала и вычисления его эффективных характеристик необходимо усреднить по полному объёму $V$ локальный закон Гука (3):

$$
\begin{gathered}
\left\langle s_{i j}\right\rangle=2 \mu_{1}\left\langle e_{i j}\right\rangle+2\left(\mu_{2}-\mu_{1}\right) c_{q}\left\langle e_{i j}\right\rangle_{q}+2\left(\mu_{p 3}-\mu_{2}\right) c_{p}\left\langle e_{i j}\right\rangle_{p}-2 \mu_{p} c_{p}\left\langle\alpha_{i j}\right\rangle_{p}, \\
\left\langle\sigma_{k k}\right\rangle=3 K_{1}\left\langle\varepsilon_{k k}\right\rangle+3\left(K_{2}-K_{1}\right) c_{q}\left\langle\varepsilon_{k k}\right\rangle_{q}+3\left(K_{p}-K_{2}\right) c_{p}\left\langle\varepsilon_{k k}\right\rangle_{p} .
\end{gathered}
$$

Соотношения (4) показывают, что для установления связи между макронапряжениями и макродеформациями необходимо выразить величины $\left\langle\varepsilon_{i j}\right\rangle_{s}$ через $\left\langle\varepsilon_{i j}\right\rangle$. Это достигается статистическим осреднением системы уравнений, состоящей из уравнений (3), уравнений равновесия $\sigma_{i j, j}(\boldsymbol{r})=0$ и формул Коши $2 \varepsilon_{i j}(\boldsymbol{r})=u_{i, j}(\boldsymbol{r})+u_{j, i}(\boldsymbol{r})$, связывающих компоненты тензора деформаций с компонентами вектора перемещений $u_{i}(\boldsymbol{r})$. Граничными условиями такой системы являются условия отсутствия фрлуктуаций величин на поверхности $S$ объема $V$.

$\mathrm{C}$ помощью тензора Грина $\mathbf{G}_{i k}(\boldsymbol{r})$ эта система уравнений сводится к системе интегральных уравнений [1]

$$
\varepsilon_{i j}^{\prime}(\boldsymbol{r})=\int_{V} \mathbf{G}_{i k, l j}\left(\boldsymbol{r}-\boldsymbol{r}_{\mathbf{1}}\right) \tau_{k l}^{\prime}\left(\boldsymbol{r}_{\mathbf{1}}\right) d \boldsymbol{r}_{\mathbf{1}} .
$$

Здесь штрихами обозначены фрлуктуации величин в объёме $V$.

Умножая уравнения (5) на $\kappa_{s}^{\prime}(\boldsymbol{r})$, усредняя их затем по объем у $V$, принимая во внимание изотропность структуры композита и используя правило механического смешивания $\langle f\rangle_{2}=\langle f\rangle_{+}$ $+c_{2}^{-1}\left\langle k^{\prime} f^{\prime}\right\rangle$, находим [1]

$$
\left\langle e_{i j}\right\rangle_{p, q}=\frac{a_{p, q}}{\Delta}\left(b_{p, q}\left\langle e_{i j}\right\rangle+d_{p, q}\left\langle\alpha_{i j}\right\rangle_{p}\right), \quad\left\langle\varepsilon_{k k}\right\rangle_{p, q}=\frac{\omega_{p, q} \eta_{p, q}}{\Delta}\left\langle\varepsilon_{k k}\right\rangle
$$

Здесь

$$
\begin{gathered}
a_{p}=\left(1+\alpha_{1}\left(m_{p}-m_{2}\right)\left(1-c_{p}\right)\right)^{-1}, \quad a_{q}=\left(1+\alpha_{1}\left(m_{2}-1\right)\left(1-c_{q}\right)\right)^{-1}, \\
\omega_{p}=\left(1+\gamma_{1}\left(q_{p}-q_{2}\right)\left(1-c_{p}\right)\right)^{-1}, \quad \omega_{q}=\left(1+\gamma_{1}\left(q_{2}-1\right)\left(1-c_{q}\right)\right)^{-1}, \\
b_{p}=1+\alpha_{1} c_{q}\left(m_{2}-1\right) a_{q}, \quad b_{q}=1+\alpha_{1} c_{p}\left(m_{p}-m_{2}\right) a_{p}, \\
\eta_{p}=1+\gamma_{1} c_{q}\left(q_{2}-1\right) \omega_{q}, \quad \eta_{q}=1+\gamma_{1} c_{p}\left(q_{p}-q_{2}\right) \omega_{p}, \\
d_{p}=\alpha_{1} m_{3}\left(1-c_{p}\right)-\alpha_{1}^{2} c_{p} c_{q} m_{p}\left(m_{2}-1\right) a_{q}, \quad d_{q}=-\alpha_{1} m_{p} c_{p}+\alpha_{1}^{2} c_{p}\left(1-c_{p}\right) m_{p}\left(m_{p}-m_{2}\right) a_{p}, \\
\alpha_{1}=\frac{2}{15} \frac{4-5 v_{1}}{1-v_{1}}, \quad \gamma_{1}=\frac{1}{3} \frac{1+v_{1}}{1-v_{1}}, \quad v_{1}=\frac{1}{2} \frac{3 K_{1}-2 \mu_{1}}{3 K_{1}+2 \mu_{1}}, \quad m_{s}=\frac{\mu_{s}}{\mu_{1}}, q_{s}=\frac{K_{s}}{K_{1}}, c_{s}=\frac{V_{s}}{V} .
\end{gathered}
$$

Подстановка формул (6) в соотношения (4) даёт макроскопический закон Гука рассматриваемой микронеоднородной среды

$$
\left\langle s_{i j}\right\rangle=2 \mu^{*}\left\langle e_{i j}\right\rangle-2 \mu^{\alpha}\left\langle\alpha_{i j}\right\rangle_{p},\left\langle\sigma_{k k}\right\rangle=3 K^{*}\left\langle\varepsilon_{k k}\right\rangle .
$$

Здесь

$$
\begin{gathered}
\mu^{*}=\mu_{1}\left(1+\frac{\left(m_{2}-1\right) c_{q} a_{q} b_{q}+\left(m_{p}-m_{2}\right) c_{p} a_{p} b_{p}}{\Delta}\right), \quad K^{*}=K_{1}\left(1+\frac{\left(q_{2}-1\right) c_{q} \omega_{q} \eta_{q}+\left(q_{p}-q_{2}\right) c_{p} \omega_{p} \eta_{p}}{\Delta}\right), \\
\mu^{\alpha}=\mu_{1}\left(c_{p} m_{p}-\frac{\left(m_{2}-1\right) c_{q} a_{q} d_{q}+\left(m_{p}-m_{2}\right) c_{p} a_{p} d_{p}}{\Delta}\right),
\end{gathered}
$$

где звёздочкой обозначены эффективные модули упругости микронеоднородной среды [1].

Для определения макроскопических условий прямого и обратного фразовых превращений в рассматриваемой среде и закона ее деформирования необходимо усреднить соотношения (2) по объему новой фазы $V_{p}$. Ограничимся здесь и далее верхней оценкой

$$
\left\langle s_{i j}\right\rangle_{p}\left\langle s_{i j}\right\rangle_{p}=\left\langle k_{+,-}^{2}(\alpha)\right\rangle_{p} .
$$

Постановка в условие (8) локального закона Гука (3) и применение правила механического смешивания дают макроскопические поверхности нагружения

$$
\left(\left\langle s_{i j}\right\rangle-2 n_{+,-}^{*}\left\langle\alpha_{i j}\right\rangle_{p}\right)\left(\left\langle s_{i j}\right\rangle-2 n_{+,-}^{*}\left\langle\alpha_{i j}\right\rangle_{p}\right)=k_{+,-}^{* 2}\left(\left\langle\alpha_{i j}\right\rangle_{p}\right)
$$


и ассоциированные с ними законы деформирования:

$$
\left\langle s_{i j}\right\rangle=k_{+,-}^{*} v_{i j}+2 n_{+,-}^{*}\left\langle\alpha_{i j}\right\rangle_{p}, \quad v_{i j}=\frac{\left\langle\dot{\alpha}_{i j}\right\rangle}{\sqrt{\left\langle\dot{\alpha}_{k l}\right\rangle\left\langle\dot{\alpha}_{k l}\right\rangle}}
$$

Здесь $k_{+,-}^{*}=k_{+,-}\left(\langle\alpha\rangle_{p}\right) \frac{\mu^{*} \Delta}{\mu_{p} a_{p} b_{p}}-$ эффрективный начальный предел фазового перехода, $n_{+,-}^{*}=\frac{k_{+,-}^{*}}{k_{+,-}} \mu_{p} \times$ $\times\left(1-a_{p} \frac{b_{p} \mu^{\alpha}+d_{p} \mu^{*}}{\mu^{*} \Delta}\right)-$ эффективный коэффициент упрочнения, характеризующий скорость перемещения поверхности (9) в шестимерном пространстве макронапряжений. Уравнения (10) описывают нелинейное деформирование среды при фазовом превращении.

Структурные средние деформации $\left\langle\alpha_{i j}\right\rangle_{p}$ необходимо выразить через объёмное содержание новой фазы $c_{p}$ и величину $\alpha_{\max }$.

В процессе фразового перехода можно выделить два этапа. На первом этапе происходит интенсивное образование зон новой фразы (зародышей), которое сопровождается быстрым ростом концентрации зародышей при относительно небольшом уровне структурных деформаций. На втором этапе - насыщении - образование новой фазы происходит в основном за счёт объёмного роста самих зародышей, внутри которых структурные деформации развиваются до своих максимальных значений. Такое деление процесса фазового превращения является достаточно условным и оба процесса на практике наблюдаются параллельно с преобладанием одного из них в разных стадиях развития уровней структурных деформаций [3]. С достаточной степенью точности этот процесс может быть описан кинетическим уравнением

$$
\frac{d \alpha}{d c_{p}}=h(1-\alpha)^{\lambda} c_{p} \quad(0 \leqslant \lambda \leqslant 1) .
$$

Параметр роста $\lambda$ уравнения (11) служит показателем разделения этапов фазового перехода. Решение уравнения (11) с учётом граничных условий $\left.c_{p}\right|_{\alpha=0}=0,\left.c_{p}\right|_{\alpha=\alpha_{\max }}=c_{2}$ даёт зависимость роста концентрации новой фразы от уровня структурных деформаций

$$
\frac{c_{p}}{c_{2}}=\sqrt{1-\left(\frac{\alpha}{\alpha_{\max }}-1\right)^{1-\lambda}} .
$$

В итоге соотношения (10) сводятся к

$$
\left\langle s_{i j}\right\rangle=\left(k_{+,-}^{*}+2 n_{+,-}^{*}\left(1-\left(1-\left(\frac{c_{p}}{c_{2}}\right)^{2}\right)^{\frac{1}{1-\lambda}}\right) \alpha_{\max }\right) v_{i j} .
$$

2. Пусть теперь фазовый переход первого рода происходит в объёме матрицы $V_{1}$ композиционного материала со сферическими включениями. Объём зарождающейся и развивающейся новой фазы внутри включений (как и в предыдущем случае) обозначим через $V_{p}$, объём старой фазы $-V_{q}\left(V_{p}+V_{q}=V_{1}\right)$. При фазовом превращении $\left(V_{p} \rightarrow V_{q}\right)$ в материале новой фазы под воздействием внешних нагрузок возникают и развиваются необратимые структурные деформации $\alpha_{i j}(\boldsymbol{r})$, вызванные перестройкой кристаллической и доменной структуры материала:

$$
\begin{aligned}
& \sigma_{i j}=2 \mu_{1} \varepsilon_{i j}+\delta_{i j} \lambda_{1} \varepsilon_{k k},\left(\boldsymbol{r} \in V_{q}\right), \sigma_{i j}=2 \mu_{2} \varepsilon_{i j}+\delta_{i j} \lambda_{2} \varepsilon_{k k},\left(\boldsymbol{r} \in V_{2}\right), \\
& \sigma_{i j}=2 \mu_{p}\left(\varepsilon_{i j}-\alpha_{i j}\right)+\delta_{i j} \lambda_{p} \varepsilon_{k k},\left(\boldsymbol{r} \in V_{p}\right) .
\end{aligned}
$$

В качестве условий фазовых переходов второго компонента в первый и обратно принимаются поверхности нагружения (2).

С помощью соответствующих индикаторных функций локальный закон Гука для среды записывается в виде

$$
\begin{gathered}
s_{i j}(\boldsymbol{r})=2\left(\mu_{1}+\left(\mu_{2}-\mu_{1}\right) \kappa_{2}(\boldsymbol{r})+\left(\mu_{p}-\mu_{1}\right) \kappa_{p}(\boldsymbol{r})\right) e_{i j}(\boldsymbol{r})-2 \mu_{p} \kappa_{p}(\boldsymbol{r}) \alpha_{i j}(\boldsymbol{r}), \\
\sigma_{k k}(\boldsymbol{r})=3\left(K_{1}+\left(K_{2}-K_{1}\right) \kappa_{2}(\boldsymbol{r})+\left(K_{p}-K_{1}\right) \kappa_{p}(\boldsymbol{r})\right) \varepsilon_{k k}(\boldsymbol{r}) .
\end{gathered}
$$

Для установления макроскопических определяющих уравнений материала и вычисления его эффективных характеристик необходимо усреднить по полному объёму $V$ локальный закон Гука (15):

$$
\begin{aligned}
& \left\langle s_{i j}\right\rangle=2 \mu_{1}\left\langle e_{i j}\right\rangle+2\left(\mu_{2}-\mu_{1}\right) c_{2}\left\langle e_{i j}\right\rangle_{2}+2\left(\mu_{p}-\mu_{1}\right) c_{p}\left\langle e_{i j}\right\rangle_{p}-2 \mu_{p} c_{p}\left\langle\alpha_{i j}\right\rangle_{p}, \\
& \left\langle\sigma_{k k}\right\rangle=3 K_{1}\left\langle\varepsilon_{k k}\right\rangle+3\left(K_{2}-K_{1}\right) c_{2}\left\langle\varepsilon_{k k}\right\rangle_{2}+3\left(K_{p}-K_{1}\right) c_{p}\left\langle\varepsilon_{k k}\right\rangle_{p} .
\end{aligned}
$$


Процедура вычисления величин $\left\langle\varepsilon_{i j}\right\rangle_{s}$, аналогичная описанной в п. 1, даёт

$$
\left\langle e_{i j}\right\rangle_{p, 2}=\frac{a_{p, 2}}{\Delta}\left(b_{p, 2}\left\langle e_{i j}\right\rangle+d_{p, 2}\left\langle\alpha_{i j}\right\rangle_{p}\right),\left\langle\varepsilon_{k k}\right\rangle_{p, 2}=\frac{\omega_{p, 2} \eta_{p, 2}}{\Delta}\left\langle\varepsilon_{k k}\right\rangle
$$

Здесь

$$
\begin{aligned}
a_{p}=\left(1+\alpha_{1}\left(m_{p}-1\right)\left(1-c_{p}\right)\right)^{-1}, & a_{2}=\left(1+\alpha_{1}\left(m_{2}-1\right)\left(1-c_{2}\right)\right)^{-1}, \\
\omega_{p}=\left(1+\gamma_{1}\left(q_{p}-1\right)\left(1-c_{p}\right)\right)^{-1}, & \omega_{2}=\left(1+\gamma_{1}\left(q_{2}-1\right)\left(1-c_{2}\right)\right)^{-1,} \\
b_{p}=1+\alpha_{1} c_{2}\left(m_{2}-1\right) a_{2}, \quad b_{2}=1+\alpha_{1} c_{p}\left(m_{p}-1\right) a_{p}, & \eta_{p}=1+\gamma_{1} c_{2}\left(q_{2}-1\right) \omega_{2}, \quad \eta_{2}=1+\gamma_{1} c_{p}\left(q_{p}-1\right) \omega_{p}, \\
d_{p}=\alpha_{1} m_{p}\left(1-c_{p}\right)-\alpha_{1}^{2} c_{p} c_{2} m_{p}\left(m_{2}-1\right) a_{2}, & d_{2}=-\alpha_{1} m_{p} c_{p}+\alpha_{1}^{2} c_{p}\left(1-c_{p}\right) m_{p}\left(m_{p}-1\right) a_{p} .
\end{aligned}
$$

Подстановка формул (17) в соотношения (16) даёт макроскопический закон Гука рассматриваемой микронеоднородной среды вида (7) со следующими эффективными модулями микронеоднородной среды:

$$
\begin{gathered}
\mu^{*}=\mu_{1}\left(1+\frac{\left(m_{2}-1\right) c_{2} a_{2} b_{2}+\left(m_{p}-1\right) c_{p} a_{p} b_{p}}{\Delta}\right), \quad K^{*}=K_{1}\left(1+\frac{\left(q_{2}-1\right) c_{2} \omega_{2} \eta_{2}+\left(m_{p}-1\right) c_{p} \omega_{p} \eta_{p}}{\Delta}\right), \\
\mu^{\alpha}=\mu_{1}\left(c_{p} m_{p}-\frac{\left(m_{2}-1\right) c_{2} a_{2} d_{2}+\left(m_{p}-1\right) c_{p} a_{p} d_{p}}{\Delta}\right) .
\end{gathered}
$$

Макроскопические поверхности выводятся как в п. 1 и имеют также вид (9), а ассоциированный с ними закон деформирования - $(10)$. Коэффрициенты $k_{+,-}^{*}$ и $n_{+,-}^{*}$ имеют тот же вид, что и в п. 1.

С помощью уравнений, аналогичных уравнениям (11), (12), можно получить

$$
\left\langle s_{i j}\right\rangle=\left(k_{+,-}^{*}+2 n_{+,-}^{*}\left(1-\left(1-\left(\frac{c_{p}}{c_{1}}\right)^{2}\right)^{\frac{1}{1-\lambda}}\right) \alpha_{\max }\right) v_{i j} .
$$

3. Пусть компоненты композиционного материала $V_{1}$ и $V_{2}$ образуют стохастическую матричную смесь. Фазовый переход первого рода происходит в объёме первого компонента $V_{1}$. Объем зарождающейся и развивающейся новой фазы внутри $V_{1}$ обозначим $V_{p}$, объём вытесняемой старой фазы $-V_{q}\left(V_{p}+V_{q}=V_{1}\right)$. При фазовом превращении $\left(V_{p} \rightarrow V_{q}\right)$ в материале новой фазы под воздействием внешних нагрузок возникают и развиваются необратимые структурные деформации $\alpha_{i j}(\boldsymbol{r})$, вызванные перестройкой кристаллической и доменной структуры материала. Закон Гука такой среды имеет вид (15), а в качестве условий фазовых переходов второго компонента в первый и обратно принимаются поверхности нагружения (2).

Локальный закон Гука для среды записывается в виде

$$
\begin{gathered}
s_{i j}(\boldsymbol{r})=2\left(\langle\mu\rangle+[\mu]_{2} \kappa_{2}^{\prime}(\boldsymbol{r})+[\mu]_{p} \kappa_{p}^{\prime}(\boldsymbol{r})\right) e_{i j}(\boldsymbol{r})-2 \mu_{p} \kappa_{p}^{\prime}(\boldsymbol{r}) \alpha_{i j}(\boldsymbol{r}), \\
\sigma_{k k}(\boldsymbol{r})=3\left(\langle K\rangle+[K]_{2} \kappa_{2}^{\prime}(\boldsymbol{r})+K_{p} \kappa_{p}^{\prime}(\boldsymbol{r})\right) \varepsilon_{k k}(\boldsymbol{r}) .
\end{gathered}
$$

Усреднённый закон Гука (19) имеет вид

$$
\left\langle s_{i j}\right\rangle=2\langle\mu\rangle\left\langle e_{i j}\right\rangle-\left\langle t_{i j}\right\rangle,\left\langle\sigma_{k k}\right\rangle=3\langle K\rangle\left\langle\varepsilon_{k k}\right\rangle-\left\langle\tau_{k k}\right\rangle .
$$

Здесь $\tau_{i j}=2 \mu_{p} \kappa_{p} \alpha_{i j}-2[\mu]_{2} \kappa_{2}^{\prime} \varepsilon_{i j}-2[\mu]_{p} \kappa_{p}^{\prime} \varepsilon_{i j}-\delta_{i j}[\lambda]_{2} \kappa_{2}^{\prime} \varepsilon_{k k}-\delta_{i j}[\lambda]_{p} \kappa_{p}^{\prime} \varepsilon_{k k}$.

Применяя к соотношению (20) процедуру вычисления величин $\left\langle\varepsilon_{i j}\right\rangle_{s}$, находим [2]

$$
\left\langle e_{i j}\right\rangle_{p, 2}=\frac{b_{p, 2}\left\langle e_{i j}\right\rangle+d_{p, 2}\left\langle\alpha_{i j}\right\rangle_{p}}{\Delta}, \quad\left\langle\varepsilon_{k k}\right\rangle_{p, 2}=\frac{\eta_{p, 2}}{\Delta}\left\langle\varepsilon_{k k}\right\rangle
$$


Здесь

$$
\begin{gathered}
b_{p}=1+\alpha[m]_{p} a_{p}\left(1-c_{p}\right)+\alpha[m]_{2} c_{2} a_{p}+\alpha^{2}[m]_{2}^{2} a_{p} a_{2} c_{2}\left(1-c_{2}\right), \\
b_{2}=1+\alpha[m]_{2} a_{2}\left(1-c_{2}\right)-\alpha[m]_{p} c_{p} a_{2}-\alpha^{2}[m]_{p}^{2} a_{p} a_{2} c_{p}\left(1-c_{p}\right), \\
\eta_{p}=1+\gamma[q]_{p} \omega_{p}\left(1-c_{p}\right)+\gamma[q]_{2} c_{2} \omega_{p}+\gamma^{2}[q]_{2}^{2} \omega_{p} \omega_{2} c_{2}\left(1-c_{2}\right), \\
\eta_{2}=1+\gamma[q]_{2} \omega_{2}\left(1-c_{2}\right)-\gamma[q]_{p} c_{p} \omega_{2}-\gamma^{2}[q]_{p}^{2} \omega_{p} \omega_{2} c_{p}\left(1-c_{p}\right), \\
d_{p}=\alpha m_{p}\left(1-c_{p}\right) a_{p}-\alpha^{2}\left(1-c_{p}\right) c_{2} m_{p}[m]_{2} a_{p} a_{2}, \quad d_{2}=-\alpha m_{p}\left(1-c_{p}\right) a_{2}-\alpha^{2}\left(1-c_{p}\right) c_{p} m_{p}[m]_{p} a_{p} a_{2}, \\
a_{p}=\left(1+\alpha[m]_{2} c_{2}+\alpha[m]_{p}\left(1-2 c_{p}\right)\right)^{-1}, \quad a_{2}=\left(1+\alpha[m]_{p} c_{p}+\alpha[m]_{2}\left(1-2 c_{2}\right)\right)^{-1} \\
\omega_{p}=\left(1+\gamma[q]_{2} c_{2}+\gamma[q]_{p}\left(1-2 c_{p}\right)\right)^{-1}, \quad \omega_{2}=\left(1+\gamma[q]_{p} c_{p}+\gamma[q]_{2}\left(1-2 c_{2}\right)\right)^{-1}, \\
\alpha=\frac{2}{15} \frac{4-5 v}{1-v}, \quad \gamma=\frac{1}{3} \frac{1+v}{1-v}, \quad v=\frac{1}{2} \frac{3\langle K\rangle-2\langle\mu\rangle}{3\langle K\rangle+2\langle\mu\rangle}, \quad \Delta=1+\alpha^{2}[m]_{p}[m]_{2} a_{p} a_{2} c_{p} c_{2} .
\end{gathered}
$$

Аналогично пп. 1-2 получаем макроскопический закон Гука рассматриваемой микронеоднородной среды в виде (7) со следующими эффективными модулями микронеоднородности:

$$
\begin{gathered}
\mu^{*}=\langle\mu\rangle\left(1+[m]_{2} c_{2}\left(\frac{b_{2}}{\Delta}-1\right)+\left[m_{p}\right] c_{p}\left(\frac{b_{p}}{\Delta}-1\right)\right), \quad \mu^{\alpha}=\langle\mu\rangle\left(c_{p} m_{p}-\frac{[m]_{2} c_{2} d_{2}+[m]_{p} c_{p} d_{p}}{\Delta}\right), \\
K^{*}=\langle K\rangle\left(1+[q]_{2} c_{2}\left(\frac{\eta_{2}}{\Delta}-1\right)+\left[q_{p}\right] c_{p}\left(\frac{\eta_{p}}{\Delta}-1\right)\right) .
\end{gathered}
$$

Для определения макроскопических условий прямого и обратного фразовых превращений в рассматриваемой среде и закона её деформирования необходимо усреднить соотношения (2) по объёму новой фазы $V_{p}$ :

$$
\left\langle s_{i j}\right\rangle_{p}\left\langle s_{i j}\right\rangle_{p}=\left\langle k_{+,-}^{2}(\alpha)\right\rangle_{p}
$$

Постановка в условие (22) локального закона Гука (19) и применение правила механического смешивания, дают макроскопические поверхности нагружения

$$
\left(\left\langle s_{i j}\right\rangle-2 n_{+,-}^{*}\left\langle\alpha_{i j}\right\rangle_{p}\right)\left(\left\langle s_{i j}\right\rangle-2 n_{+,-}^{*}\left\langle\alpha_{i j}\right\rangle_{p}\right)=k_{+,-}^{* 2}\left(\left\langle\alpha_{i j}\right\rangle_{p}\right)
$$

и ассоциированный с ними закон деформирования

$$
\left\langle s_{i j}\right\rangle=k_{+,-}^{*} v_{i j}+2 n_{+,-}^{*}\left\langle\alpha_{i j}\right\rangle_{p} .
$$

Здесь $k_{+,-}^{*}=k_{+,-}\left(\langle\alpha\rangle_{p}\right) \frac{\mu^{*} \Delta}{\mu_{p} b_{p}}-$ эффрективный начальный предел фазового перехода, $n_{+,-}^{*}=\frac{k_{+,-}^{*}}{k_{+,-}} \mu_{p} \times$ $\times\left(1-a_{p} \frac{b_{p} \mu^{\alpha}+d_{p} \mu^{*}}{\mu^{*} \Delta}\right)-$ эфффективный коэффициент упрочнения, характеризующий скорость перемещения поверхности (23) в шестимерном пространстве макронапряжений.

Структурные средние деформации $\left\langle\alpha_{i j}\right\rangle_{p}$ выражаются через объёмное содержание новой фазы $c_{p}$ и величину $\alpha_{\max }$ формулой $\frac{c_{p}}{c_{1}}=\sqrt{1-\left(\frac{\left\langle\alpha_{i j}\right\rangle_{p}}{\alpha_{\max }}-1\right)^{1-\lambda}}$.

Соотношение (24) принимает вид

$$
\left\langle s_{i j}\right\rangle=\left(k_{+,-}^{*}+2 n_{+,-}^{*}\left(1-\left(1-\left(\frac{c_{p}}{c_{1}}\right)^{2}\right)^{\frac{1}{1-\lambda}}\right) \alpha_{\max }\right) v_{i j} .
$$

\section{БИБЛИОГРАФИЧЕСКИЙ СПИСОК}

1. Ильина, E.A. К теории сверхупругого поведения композиционных материалов с нестабильными компонентами [Текст]: Е.А. Ильина, А. Г. Михеев, Л. А. Сараев // Вестн. Сам. госуд. техн. ун-та. Сер.: Физ.-мат. науки. 2004. - № 26. - C. 108-114. - ISBN 5-7964-0515-2.

2. Сараев, Л.А. Моделирование макроскопических пластических свойств многокомпонентных композиционных материалов [Текст] / Л. А. Сараев. - Самара: СамГУ, 2000. - 182 с.

3. Физическое металловедение (в 3-х томах) [Текст] / Под ред. Р. Кана. - М.: Мир. - Т. 2. - 1968. - 490 с. 